\title{
The role of EGF-R expression on patient survival in lung cancer: a systematic review with meta-analysis
}

\author{
A-P. Meert*, B. Martin*, P. Delmotte*, T. Berghmans*, J-J. Lafitte ${ }^{\#}$, C. Mascaux*, M. Paesmans", \\ E. Steels*, J-M. Verdebout ${ }^{+}$, J-P. Sculier*
}

The role of EGF-R expression on patient survival in lung cancer: a systematic review with meta-analysis. A-P. Meert, B. Martin, P. Delmotte, T. Berghmans, J-J. Lafitte, C. Mascaux, M. Paesmans, E. Steels, J-M. Verdebout, J-P. Sculier. (C) ERS Journals Ltd 2002.

ABSTRACT: The prognostic value of epidermal growth factor receptor (EGF-R) for survival of patients with lung cancer remains controversial. The authors performed a systematic review of the literature in order to clarify its impact.

Published studies were identified using an electronic search in order to aggregate the available survival results, after a methodological assessment using a scale specifically designed by the European Lung Cancer Working Party (ELCWP). To be eligible, a study had to have dealt with EGF-R assessment in lung cancer patients on the primary site and to have analysed survival according to EGF-R expression.

Among the 16 eligible studies, 14 assessed any nonsmall-cell lung cancer (NSCLC) subtype, one adenocarcinoma only and one squamous-cell carcinoma only. The overall median quality score was $\mathbf{5 6 . 3} \%$, with no significant difference either between studies assessable or not assessable for meta-analysis or between studies with significant and nonsignificant results. One individual trial reported a survival benefit for patients with EGF-R expression, three a survival disadvantage and 12 no statistically significant difference. Eleven studies $(2,185$ patients) provided sufficient data to allow a metaanalysis of the survival results. EGF-R expression positivity was determined according to the cut-off as determined by the authors. The meta-analysis showed that EGF-R expression was not a statistically significant prognostic factor for survival in NSCLC. In the subgroup of studies using immunohistochemistry, statistical tests reached a significant level against EGF-R.

Epidermal growth factor receptor might be a poor prognostic factor for survival in nonsmall-cell lung cancer. The amplitude of the impact is small, however, and may be subject to publication bias.

Eur Respir J 2002; 20: 975-981.
*Dept of Internal Medicine, "Data Centre, and ${ }^{+}$Service of Pathology, Jules Bordet Institute, Brussels, Belgium. ${ }^{\#}$ Service of Pneumology and Thoracic Medicine, CHU Calmette, Lille, France.

Correspondence: A-P. Meert

Institut Jules Bordet

1, rue Héger Bordet

1000-Bruxelles

Belgium

Fax: 3225343756

E-mail: ap.meert@bordet.be

Keywords: Epidermal growth factor receptor

lung cancer

systematic review

Received: November 192001

Accepted after revision: June 182002

This study was supported by an FNRSTélévie grant (7.4512.98), Belgium. A-P. Meert is an FNRS research fellow.
Lung cancer is the most common cause of death by malignancy in industrialised countries and $<15 \%$ of patients can be cured and enjoy long-term survival. This poor prognosis can, however, be modulated by characteristics related to the patient or the tumour. These prognostic factors can be used for different purposes, such as a better understanding of the natural history of the disease, the identification of homogeneous patient populations with a similar outcome profile and the prediction of response (or not) to treatment. Some independent clinical and biological predictors of survival have been identified [1] for resectable nonsmall-cell lung cancer (NSCLC): age, performance status and tumour node metastasis (TNM) stage [2]. In small-cell lung cancer, only disease stage and performance status have been shown to consistently predict outcome [1]. Among routine biological factors, serum lactate dehydrogenase, white blood cell and neutrophil counts have been shown to significantly predict survival in NSCLC [3].

Recent developments in cytogenetic and molecular biology have provided new ways to analyse prognosis. Biological substaging, using molecular markers, in a risk stratification strategy has been proposed. Tumour suppressor genes, proto-oncogenes and markers of metastatic propensity and proliferation are some of the different research tools. Among them, the epidermal growth factor (EGF) family plays an important role in local tumour growth. This phenomenon requires growth-regulating proteins, such as EGF receptor (EGF-R) or proto-oncogene erb-B2. The specific EGF-R, first purified from the A431 cell line, is the protein product of the oncogene HER1. It is a $170 \mathrm{kDa}$ transmembrane glycoprotein (a member of the erbB family of cell surface receptors) that is composed of three major regions: 1) a N-terminus extracellular ligand-binding area; 2) a hydrophobic transmembrane domain; and 3) a C-terminus intracellular region with tyrosine kinase activity. The extracellular domain is a ligand-binding site for various polypeptide growth factors: EGF, transforming growth factor- $\alpha$ (TGF- $\alpha)$, amphiregulin, betacellulin, 
heparin-binding protein, epiregulin and vaccinian virus growth factor. The binding of one of these ligands to the extracellular region of EGF-R implies a dimerisation of EGF-R, resulting in autophosphorylation and activation of cytoplasmic signal proteins that are involved in transmitting a mitogenic signal [4]. EGF$\mathrm{R}$ also plays a role in cell motility, adhesion (through regulation of the interaction of E-cadherin with the actin cytoskeleton), invasion and angiogenesis [5]. EGF-R is found on the surface of many cells including normal and malignant cells of epidermal or mesenchymal origin. EGF-R is expressed or overexpressed in a wide variety of solid human tumours, including NSCLC, prostate, breast, gastric, colorectal, head and neck, bladder, ovarian cancers and glioblastoma.

EGF-R is expressed poorly or not expressed at all in normal lung tissue and in small-cell lung cancer. There is increased expression in preneoplastic lesions (metaplasia/dysplasia). In NSCLC, EGF-R overexpression is reported in $13-80 \%$ of tumours $(24-89 \%$ of squamous-cell lung cancer and $23-46 \%$ of adenocarcinoma). EGF-R gene amplification occurs in $\sim 6 \%$ of all NSCLC and $28 \%$ of squamous-cell lung cancers. Finally, in NSCLC, a mutation called EGF-RvIII, containing a constitutively active tyrosine kinase, the activation of which is independent of ligand binding, was observed. Numerous studies have suggested that expression of high levels of EGF-R is associated with advanced or metastatic disease and a poor prognosis. Unfortunately, the reports about its relationship with survival have provided inconsistent results in lung cancer. Therefore, the current authors have performed a systematic review of the literature to assess the prognostic value of EGF-R overexpression on survival in patients with lung cancer.

\section{Materials and methods}

\section{Publication selection}

To be eligible for this review, trials had to deal with lung cancer only, to evaluate the correlation of EGF$\mathrm{R}$ status and survival, to measure EGF-R expression in the primary tumour (not in metastatic tissue or in tissue adjacent to the tumour) and to be published as a full paper in English or French language peerreviewed literature.

Articles were identified by an electronic search on Medline using the keywords "lung neoplasms", "EGF", "EGF-R", "c-erb-B1" and "epidermal growth factor". The references reported in all the identified studies were used for completion of the trial's search. When authors reported on the same patient populations in several publications, only the most recent or complete study was included into the analysis, in order to avoid overlap between cohorts. The search ended in July 2001.

\section{Methodological assessment}

In order to assess the methodology, each trial was read and scored according to the European Lung
Cancer Working Party (ELCWP) scale by 10 investigators (including seven physicians, one pathologist, one biologist and one biostatistician). The scoring system used in this literature review has already been described in one of the authors' prior systematic reviews [6]. Each item of the score was quoted using an ordinal scale (possible values $2,1,0$ ) by consensus in meetings where at least two thirds of the investigators needed to be present. The participation of many readers was a guarantee for the correct interpretation of the articles. The overall score assessed many dimensions of methodology, grouped into four main categories: 1) the scientific design; 2) the description of the laboratory methods used to identify the presence of EGF-R; 3) the generalisability of the results; and 4) the analysis of the study data. Each category had a maximal score of 10 points with an overall maximum theoretical score of 40 points. When an item was not applicable to a study, its value was not taken into account in the total for the category. The final scores were expressed as percentages, with higher values reflecting a better methodological quality. Studies included in the systematic review were called "eligible" and those providing sufficient data for the metaanalysis "evaluable".

\section{Statistical method}

A study was considered significant if the p-value for the statistical test comparing survival distributions between the groups with and without EGF-R expression was $<0.05$. The study was called "positive" when EGF-R expression was identified as a significant favourable prognostic factor for survival. The study was called "negative" if EGF-R expression was associated with a significant detrimental effect on survival. Finally, a study was called "not significant" if no difference between the groups expressing EGF-R or not expressing EGF-R was detected. The association between two continuous variables, such as the quality score, was measured by the Spearman rank correlation coefficient. Nonparametric tests were used to compare the distribution of the quality scores according to the value of a discrete variable (Mann-Whitney tests for dichotomic variables or Kruskal-Wallis tests for multiple class variables).

For the quantitative aggregation of survival results, the impact of EGF-R expression on survival was measured by the hazard ratio (HR) between the two survival distributions. For each trial, this HR was estimated by a method depending on the data provided in the publications. The most accurate method consisted of calculating the estimated HR and its SE from the reported results or calculating them directly using two of the following parameters: the confidence interval (CI) for the HR, the log-rank statistic, its pvalue or the O-E statistic (difference between numbers of observed and expected events). If those data were not available, the following were studied: the total number of events, the number of patients at risk in each group and the logrank statistic or its p-value, allowing calculation of an approximation of the HR estimate. Finally, if the only available data were in the 
form of graphical representations of the survival distributions, survival rates were extracted at specified times in order to reconstruct the HR estimate and its variance, with the assumption that the rate at which patients were censored was constant during the study follow-up [7]. The individual $\mathrm{HR}$ estimates were combined into an overall HR using Peto's method [8], which consisted of a fixed effect model assuming homogeneity of the individual true HRs. This assumption was tested by performing Chi-squared tests for heterogeneity. If the assumption of homogeneity had to be rejected, a random-effects model was used as a second stage. By convention, an observed HR of $>1$ implied a worse survival for the group with positive EGF-R expression. This pejorative impact of EGF-R on survival was considered as statistically significant if the $95 \%$ CI for the overall HR did not overlap 1.

\section{Results}

\section{Study selection and characteristics}

At the time of analysis, 21 studies published between 1989-2001 were selected [9-29]. Five of the articles were excluded because an identical cohort of patients was used in other selected publications (studies excluded [15, 25-28]; studies included [19, 20, 22, 29]).

The total number of patients included was 2,810 , ranging from 19-505 patients by trial. The main characteristics of the 16 eligible studies are shown in table 1. Fourteen dealt with NSCLC of all histological subtypes, one with adenocarcinoma only and one with squamous-cell carcinoma only. Patients treated by surgery for locoregional disease (stages I-IIIB) were included in 11 studies. Immunohistochemistry was the technique most frequently used to detect EGF-R expression (13 trials). In one trial, authors used immunohistochemistry and Northern blot; in another, the concentration of EGF-R was measured by competitive ligand-binding technique using a radio-iodine labelled EGF; and in the last study, EGF-R was detected by polymerase chain reaction. Different antibodies were used to assess EGF-R expression by immunohistochemistry: three used an anti-EGFR mouse monoclonal antibody (Triton, Alameda, CA, USA); two used clone EGFR (113; Novocastra, Newcastle, UK); two used personal EGF-R 1 murine monoclonal antobody; one used EGF-R 1 product (Amersham, Buckinghamshire, UK); one used rabbit polyclonal antibody EGF-R Ab-4 (Dianova, Hamburg, Germany); one used anti-EGF-R mouse serum (Transformation Research Inc., Framingham, MA, USA); one used EGF-R monoclonal (014H4819; Sigma, St Louis, MO, USA) and one used personal monoclonal antibody F4. In most cases, assessment of EGF-R status was performed on surgical samples.

\section{Study results}

According to EGF-R survival prognostic analysis, three studies were negative, one was positive and 12 were nonsignificant, of which only 11 were evaluable for the meta-analysis. Considered separately, the study of adenocarcinoma showed no advantages or disadvantages in terms of survival when EGF-R was expressed, and in the squamous carcinoma study, EGF-R expression seemed to suggest a worse prognosis.

Overall, according to the positivity threshold for EGF-R expression, as defined by the study's authors, EGF-R was expressed in $51.1 \%$ of the evaluable patients, in $46.2 \%$ in the study concerning adenocarcinoma alone and in $82.6 \%$ in the study of squamouscell carcinoma alone.

EGF-R expression was found in $50.0 \%, 53.8 \%$ and $48.9 \%$ of patients presenting with stages I, I-III or I-IV, respectively.

Table 1.-Main characteristics and results of the eligible studies

\begin{tabular}{|c|c|c|c|c|c|c|c|}
\hline $\begin{array}{l}\text { First author } \\
\text { [Ref. no.] }\end{array}$ & Year & Histology & Stage & $\mathrm{N}$ pts & Technique & HR estimation & Results \\
\hline DAZZI [18] & 1989 & NSCLC & $\mathrm{I}-?$ & 152 & IHC & No data & NS \\
\hline Veale [23] & 1993 & NSCLC & I-III & 19 & Ligand competition & $\mathrm{HR}+\mathrm{CI} 95 \%$ & Negative \\
\hline TATEISHI [29] & 1994 & Adenocarcinoma & I-IV & 119 & $\mathrm{IHC}$ & Survival curves & NS \\
\hline GIATROMANOLAKI [11] & 1996 & NSCLC & I-II & 107 & $\mathrm{IHC}$ & No data & NS \\
\hline KOUKOURAKIS [19] & 1997 & NSCLC & I-II & 107 & $\mathrm{IHC}$ & No data & NS \\
\hline PASTORINO [14] & 1997 & NSCLC & I & 505 & $\mathrm{IHC}$ & $\mathrm{HR}+\mathrm{CI} 95 \%$ & NS \\
\hline RuSCH [22] & 1997 & NSCLC & I-IIIA & 96 & Northern+IHC & Survival curves & Positive \\
\hline GREATENS [24] & 1998 & NSCLC & I-IV & 101 & IHC & No data & NS \\
\hline PFEIFFER [17] & 1998 & NSCLC & I-IV & 180 & $\mathrm{IHC}$ & Survival curves & NS \\
\hline VoLM [20] & 1998 & Squamous & I-III & 121 & $\mathrm{IHC}$ & Survival curves & Negative \\
\hline FONTANINI [21] & 1998 & NSCLC & I-IIIA & 195 & $\mathrm{IHC}$ & Logrank $+\mathrm{N}$ events & NS \\
\hline FU [12] & 1999 & NSCLC & I-IIIB & 158 & $\mathrm{IHC}$ & No data & NS \\
\hline D'AMICO [13] & 1999 & NSCLC & I & 408 & $\mathrm{IHC}$ & Logrank $+\mathrm{N}$ events & NS \\
\hline OHSAKI [16] & 2000 & NSCLC & I-IV & 290 & $\mathrm{IHC}$ & Survival curves & Negative \\
\hline Cox [9] & 2000 & NSCLC & I-IIIA & 169 & IHC & Logrank $+\mathrm{N}$ events & NS \\
\hline BRABENDER [10] & 2001 & NSCLC & I-IIIA & 83 & PCR & $\mathrm{HR}+\mathrm{CI} 95 \%$ & NS \\
\hline
\end{tabular}

$\mathrm{N}$ pts: number of patients; HR: hazard ratio; NSCLC: nonsmall-cell lung cancer; IHC: immunohistochemistry; PCR: polymerase chain reaction; $\mathrm{CI}$ : confidence interval; NS: nonsignificant. 


\section{Quality assessment}

The overall quality score ranged from $25.8-70.7 \%$, with a median of $56.3 \%$. The "design" subscore had the lowest value (four out of 10), except for the nonevaluable studies where the "analysis results" subscore had the lowest value (table 2). There was no statistically significant quality difference between evaluable and nonevaluable studies for meta-analysis (median overall score: 61.7 versus $48.8 \%, \mathrm{p}=0.19$ ). For the 11 trials evaluable for meta-analysis, the overall quality score ranged from $44.1-70.7 \%$ with a median of $61.7 \%$. The most poorly described items $(<30 \%$ of the maximum theoretical score) were the initial work-up of the disease itself and the number of inaccessible samples with a description of exclusion causes. No statistically significant quality difference was shown between the four significant trials and the 12 nonsignificant trials (median overall scores 54.7 versus $56.3 \%, \mathrm{p}=0.9)$. There was no difference between the significant and nonsignificant studies evaluable for the meta-analysis (54.7 versus $61.7 \%, \mathrm{p}=0.44$ ).

\section{Meta-analysis}

The absence of a significant quality difference between significant and nonsignificant studies made it possible to perform a quantitative aggregation of the survival results. Among the 16 trials eligible for the systematic review, five could not be included in the meta-analysis (625 patients) due to insufficient data to estimate the HR. Consequently, the analysis was restricted to 11 studies and 2,185 patients.

The HRs of the 11 evaluable studies were calculated by one of the three methods reported in the Materials and methods section. HRs and 95\% confidence intervals (CIs) were published in three trials. They were approximated from the log-rank statistic and the number of events in three studies. Finally, the HR and its variability had to be extrapolated from the graphical representations of the survival distributions in five others.

The test of heterogeneity was significant $(\mathrm{p}=0.02)$.

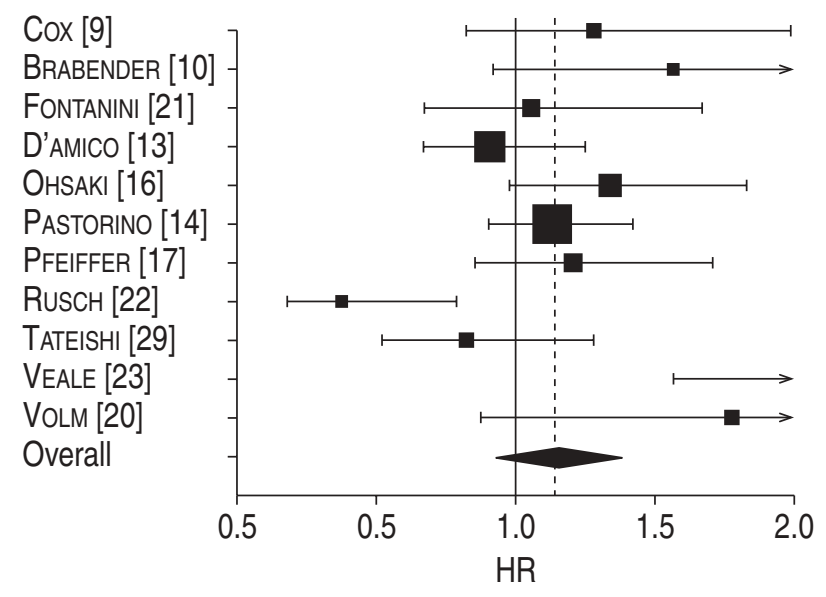

Fig. 1.- Results of the meta-analysis of all the studies. A hazard ratio (HR) of $<1(-)$ implies a survival benefit for the group with epidermal growth factor receptor expression. The square size is proportional to the number of patients included in each study. The centre of the lozenge gives the combined HR for the metaanalysis and its extremities the $95 \%$ confidence interval.

Thus, the HR was calculated using a random-effects model and a value was obtained that was not statistically significant (HR 1.14, 95\% CI 0.94-1.39) (fig. 1). When only the eight evaluable studies using immunohistochemistry were considered, there was no more heterogeneity $(\mathrm{p}=0.45)$. The result of metaanalysis was just significant (HR 1.13, 95\% CI 1.00-1.28) (fig. 2), meaning that patients with a tumour not expressing EGF-R had a better survival.

\section{Discussion}

In this systematic review, which pooled all the studies comparing the survival of lung cancer patients according to the tumour expression of EGF-R, EGF-R presence seems to be a factor for a poor prognosis for survival in NSCLC. Statistical significance is reached when immunohistochemistry is used to detect its expression. This observation is potentially

Table 2.-Quality scores analysis of the eligible studies

\begin{tabular}{|c|c|c|c|c|c|}
\hline Studies (n) & Design $^{\#}$ & $\begin{array}{l}\text { Laboratory } \\
\text { method }^{\#}\end{array}$ & Generalisability $^{\#}$ & $\begin{array}{l}\text { Results } \\
\text { analysis }{ }^{\#}\end{array}$ & $\begin{array}{l}\text { Global } \\
\text { score } \%\end{array}$ \\
\hline All (16) & 4.00 & 6.43 & 6.67 & 5.00 & 56.34 \\
\hline Evaluable for MA (11) & 4.00 & 7.14 & 6.67 & 5.00 & 61.70 \\
\hline $\begin{array}{l}\text { Not evaluable for } \\
\text { MA (5) }\end{array}$ & 4.00 & 5.00 & 5.00 & 3.75 & 48.84 \\
\hline p-value & 0.28 & 0.11 & 0.61 & 0.14 & 0.19 \\
\hline Significant (4) & 5.00 & 6.43 & 5.42 & 5.00 & 54.70 \\
\hline Nonsignificant (12) & 4.00 & 6.43 & 6.67 & 5.62 & 56.34 \\
\hline p-value & 0.36 & 0.85 & 0.27 & 0.95 & 0.90 \\
\hline Negative (3) & 4.00 & 5.71 & 4.17 & 5.00 & 47.38 \\
\hline $\begin{array}{l}\text { Significant studies in } \\
\text { MA (4) }\end{array}$ & 5.00 & 6.42 & 5.41 & 5.00 & 54.70 \\
\hline $\begin{array}{l}\text { Nonsignificant studies } \\
\text { in MA (7) }\end{array}$ & 4.00 & 7.14 & 6.67 & 7.50 & 61.70 \\
\hline p-value & 0.57 & 0.70 & 0.13 & 0.57 & 0.44 \\
\hline
\end{tabular}

Scores are median scores of the studies. MA: meta-analysis. ${ }^{\#}$ : scored out of 10 . 


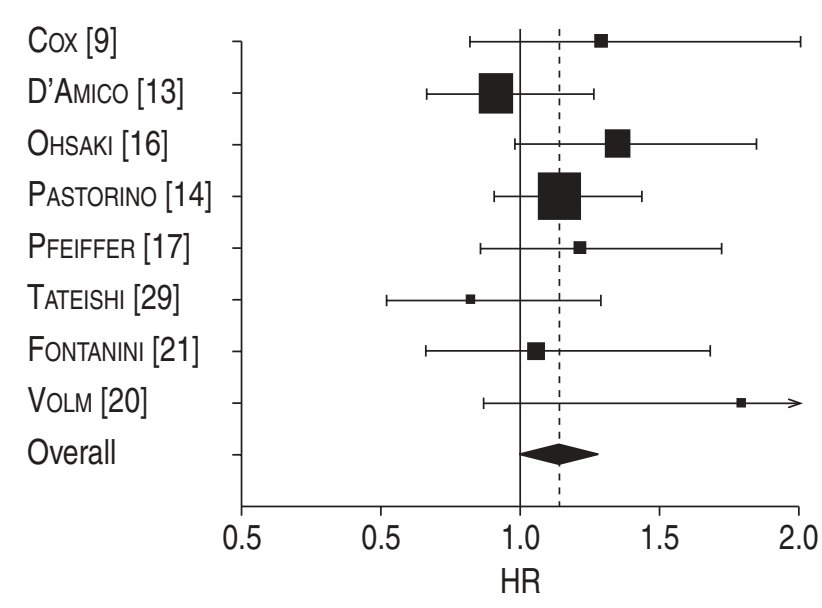

Fig. 2. - Results of the meta-analysis of the studies using immunohistochemistry to detect epidermal growth factor receptor (EGF$\mathrm{R})$ expression in nonsmall-cell lung cancer. A hazard ratio (HR) of $<1 \quad(-)$ implies a survival benefit for the group with EGF-R expression. The square size is proportional to the number of patients included in each study. The centre of the lozenge gives the combined HR for the meta-analysis and its extremities the $95 \%$ confidence interval.

important for prognostic reasons and treatment purposes, in addition to improving the understanding of lung cancer biology. Identification of prognostic factors allows the definition of high-risk groups of patients for whom specific therapy might be necessary, or a stratification has to be performed in controlled trials. Moreover, EGF-R may be a potential therapeutic target. Interruption of the signalling pathways for EGF-R can be achieved using antibodies (e.g. IMC-225) or small-molecule, orally-active, tyrosine kinase inhibitors (e.g. ZD1839). Both approaches demonstrate activity and may provide a future treatment for NSCLC.

The biological role of EGF-R can explain its poor prognosis. Its expression is generally low in normal lung tissue and is only detected in the basal layer of the bronchial epithelium. EGF-R expression is enhanced in metaplastic, preneoplastic and neoplastic lesions with a progressively increased intensity of staining. In these lesions, EGF-R staining is also present in the superficial layers of the bronchial epithelium [30]. Therefore, it has been proposed as an early marker of neoplastic transformation. In NSCLC, EGF-R and its most important ligand, TGF- $\alpha$ are thought to act by a paracrine and/or autocrine loop.

The current authors decided to perform a metaanalysis on EGF-R expression in lung cancer after assessment of the methodological quality of the publications to avoid biases due to differences in quality between positive and negative studies. A methodology that is similar to previous systematic reviews by the current authors' group on the treatment of lung cancer [31] was used but also adapted to the field of biological prognostic factors [6]. The absence of a statistically significant difference in quality score between the significant and nonsignificant publications allowed the authors to perform a quantitative aggregation (meta-analysis) of the results of the individual trials. The statistical absence of difference between the publications could not be excluded because of the small number of studies.

However, this approach does not prevent all potential bias. The review was restricted to articles published in English or French because other languages, such as Japanese, were not accessible for the readers. This selection could favour the positive studies, as positive studies are often published in English, while negative studies tend to be reported in native languages [32].

Another possible source of confusion is the use of the same cohort of patients in different publications. It might be difficult to avoid the same patients being included more than once in the meta-analysis, although publications where this seemed to be the case were excluded. The current authors have assumed that authors have been honest and have not reported the results from the same cohort of patients without mentioning it in their publication.

Five studies were excluded from the meta-analysis due to lack of the data necessary for aggregation. There is therefore a publication bias. These studies were not all statistically significant. It is known that this type of study is less frequently published or, if they are, with less detailed results, making them less assessable.

The method of extrapolation of HRs also needs to be discussed. When HRs were not reported by the authors (seven studies), they were calculated from the data available in the article and, if this was not possible, were extrapolated from the survival curves, implying assumptions on the censoring process. This approach might also have been associated with errors due to imprecision of the reading, although three independent people read the curves to diminish the reading variation.

The techniques used to detect EGF-R expression might also be potential sources of bias. Immunohistochemistry was the most frequent technique used. Immunohistochemical results depend on the primary antibody used. In this review, a range of antibodies were used to detect the protein. The dilution of the antibody also differed, leading to a potential problem because the sensitivity of the method can depend on the antibody concentration. Due to the small groups of studies evaluating the same antibody, subgroup analysis could not be performed to explore this technical problem. Immunohistochemical results also depend on the type of tissue conservation (fixed or frozen) and on the antigen-retrieval technique. Moreover, this technique remains mainly qualitative with some subjectivity in the assessment. In a previous study [27], Rusch et al. [27] showed that only uniform and intense levels of immunohistochemical staining in the primary tumour correlated with overexpression at the ribonucleic acid (RNA) level compared to the uninvolved lung; less intense staining or specific staining in few cells did not correlate as highly with overexpression at the total cellular RNA level. The cut-off in the number of positive cells defining an EGF-R-positive tumour is also often arbitrary and varies according to the investigators, from a few per cent to $>50 \%$. The use of different cut-off points for 
immunohistochemistry is of critical importance [33]. The choice of a cut-off is often arbitrary, although the selection of the median value of the expression levels is a standard approach to analysis of new prognostic factors, even if it may lead to some loss of information. An optimal threshold needs to be defined for EGF-R. Therefore, molecular biology techniques are actually necessary in order to validate the immunohistochemistry results. Conversely, the advantages of immunohistochemistry are the maintenance of the tissue architecture, the possibility of localising the antigen and the fact that it is probably the most applicable and cost-effective technique for routine use. Radioligand binding analysis is more quantitative but may involve examination of noncancerous stroma cell, in contrast to immunohistochemistry. A highly significant correlation between results obtained by enzyme-linked immunosorbent assay (ELISA) and immunohistochemistry for the measurement of EGF-R was obtained by PFEIFFER et al. [17]. The diadvantages of ELISA are the requirement of fresh or frozen tissue and the need to calculate an average EGF-R content of tumour and normal tissue. Most studies have thus used different techniques to detect EGF-R expression. Standardisation and validation of the immunohistochemical analyses, probably by comparison with a molecular biology technique, is therefore important in order to correctly interpret research results and to have a reproducible routine test.

Finally, the test for heterogeneity between the studies did not remain significant when only the trials evaluating EGF-R by immunohistochemistry were considered, while it reached significance when all types of studies were considered. However, by using a random-effects model, which took heterogeneity into account, the issue of heterogeneity among the individual trials could be addressed and the source of heterogeneity could be explored. It should be noted that a previous study has shown that immunohistochemical staining in NSCLC correlates with overexpression at the level of total cellular RNA [27], supporting the use of this technique.

This review took into account only fully published studies. The authors did not look for unpublished trials and abstracts because fully published data was required. Meta-analysis based on individual data is considered by some authors as a gold standard [34]. Systematic review of the literature should not be confused with meta-analysis of individual patient data. The first approach is only based on fully published studies and provides an exhaustive and critical analysis of the topic with an adequate methodology based on the criteria of Mulnow [35] and with data aggregation when possible. The second approach is a new study taking into account all performed trials on the topic, published or not, requiring individual data update by the investigators. Nevertheless, as shown by the meta-analysis of prophylactic cerebral irradiation in small-cell lung cancer [36], based on published data, the current approach allows the same results as those obtained in meta-analysis based on individual data to be found [37]. This experience supports the validity of the current approach. In addition, this review deals with prognostic factor studies and, as they are more often retrospective, it is much more difficult to identify unpublished data than for clinical trials data.

In conclusion, this systematic review suggests that epidermal growth factor receptor expression might be a poor prognostic factor for survival in patients with nonsmall-cell lung cancer, if detected by immunochemistry. The results were based on an aggregation of data obtained by univariate survival analysis in retrospective trials. In order to become a useful prognostic factor at the level of individual patients and in the context of targeted therapy, these results need to be confirmed by an adequately designed prospective study and the exact value of epidermal growth factor receptor expression needs to be determined by an appropriate multivariate analysis taking into account the classical well-defined prognostic factors for lung cancer.

\section{References}

1. Paesmans M, Sculier JP. Prognosis factors in lung cancer (Facteurs pronostiques des cancers bronchopulmonaires). Arnette 1998; 239-247.

2. Strauss GM. Prognostic markers in resectable nonsmall cell lung cancer. Oncol Clin North Am 1997; 11: 409-434.

3. Kanters SD, Lammers JW, Voest EE. Molecular and biological factors in the prognosis of non-small cell lung cancer. Eur Respir J 1995; 8: 1389-1397.

4. Prigent SA, Lemoine NR. The type 1 (EGFR-related) family of growth factor receptors and their ligands. Prog Growth Factor Res 1992; 4: 1-24.

5. de Jong JS, van Diest PJ, van d Valk P, Baak JP. Expression of growth factors, growth-inhibiting factors, and their receptors in invasive breast cancer. II: Correlations with proliferation and angiogenesis. J Pathol 1998; 184: 53-57.

6. Steels E, Paesmans M, Berghmans T, et al. Role of p53 on the survival of patients with lung cancer as assessed by systematic review of the literature. Eur Respir $J$ 2001; 18: 705-719.

7. Parmar MK, Torri V, Stewart L. Extracting summary statistics to perform meta-analyses of the published literature for survival endpoints. Stat Med 1998; 17: 2815-2834.

8. Yusuf S, Peto R, Lewis J, Collins R, Sleight P. Beta blockade during and after myocardial infarction: an overview of the randomized trials. Prog Cardiovasc Dis 1985; 27: 335-371.

9. Cox G, Jones JL, O'Byrne KJ. Matrix metalloproteinase 9 and the epidermal growth factor signal pathway in operable non-small cell lung cancer. Clin Cancer Res 2000; 6: 2349-2355.

10. Brabender J, Danenberg KD, Metzger R, et al. Epidermal growth factor receptor and her2-neu mRNA expression in non small cell lung cancer is correlated with survival. Clin Cancer Res 2001; 7: $1850-1855$

11. Giatromanolaki A, Gorgoulis V, Chetty $\mathrm{R}$, et al. C-erbB-2 oncoprotein expression in operable non-small cell lung cancer. Anticancer Res 1996; 16: 987-993.

12. Fu X-L, Zhu X-Z, Shi D-R, et al. Study of prognostic predictors for non-small cell lung cancer. Lung Cancer 1999; 23: 143-152. 
13. D'Amico TA, Massey M, Herndon JE, Moore M-B, Harpole DH. A biologic risk model for stage I lung cancer: immunohistochemical analysis of 408 patients with the use of ten molecular markers. $J$ Thor Cardiovasc Surg 1999; 117: 736-743.

14. Pastorino U, Andreola S, Tagliabue E, et al. Immunocytochemical markers in stage I lung cancer: relevance to prognosis. J Clin Oncology 1997; 15: $2858-2865$.

15. Tateishi M, Ishida $\mathrm{T}$, Mitsudomi $\mathrm{T}$, Kaneko $\mathrm{S}$, Sugimachi K. Immunohistochemical evidence of autocrine growth factors in adenocarcinoma of the human lung. Cancer Res 1990; 50: 7077-7080.

16. Ohsaki Y, Tanno S, Fujita Y, et al. Epidermal growth factor receptor expression correlates with poor prognosis in non-small cell lung cancer patients with p53 overexpression. Oncology Rep 2000; 7: 603607.

17. Pfeiffer P, Nexo E, Bentzen SM, Clausen PP, Andersen K, Rose C. Enzyme-linked immunosorbent assay of epidermal growth factor receptor in lung cancer: comparisons with immunohistochemistry, clinicopathological features and prognosis. $\mathrm{Br} J$ Cancer 1998; 78: 96-99.

18. Dazzi H, Hasleton PS, Thatcher N, et al. Expression of epidermal growth factor receptor (EGF-R) in nonsmall cell lung cancer. Use of archival tissue and correlation of EGF-R with histology, tumour size, node status and survival. Br J Cancer 1989; 59: 746749 .

19. Koukourakis MI, Giatromanolaki A, O'Byrne KJ, et al. Potential role of bcl-2 as a suppressor of tumour angiogenesis in non-small-cell lung cancer. Int $J$ Cancer 1997; 74: 565-570.

20. Volm M, Rittgen W, Drings P. Prognostic value of ERBB-1, VEGF, cyclin A, FOS, JUN and MYC in patients with squamous cell lung carcinomas. $\mathrm{Br}$ J Cancer 1998; 77: 663-669.

21. Fontanini G, De Laurentis M, Vignati S, et al. Evaluation of epidermal growth factor-related growth factors and receptors and of neoangiogenesis in completely resected stage I-IIIA non-small-cell lung cancer: amphiregulin and microvessel count are independent prognostic indicators of survival. Clin Cancer Res 1998; 4: 241-249.

22. Rusch V, Klimstra D, Venkatraman E, Pisters PWT, Langenfeld J, Dmitrovsky E. Overexpression of the epidermal growth factor receptor and its ligand transforming growth factor $\alpha$ is frequent in resectable non-small cell lung cancer but does not predict tumor progression. Clin Cancer Res 1997; 3: 515-522.

23. Veale D, Kerr N, Gibson GJ, Kelly PJ, Harris AL. The relationship of quantitative epidermal growth factor receptor expression in non-small cell lung cancer to long term survival. Br J Cancer 1993; 68: $162-165$.

24. Greatens TM, Niehans GA, Rubins JB, et al. Do molecular markers predict survival in non-small-cell lung cancer? Am J Respir Crit Care Med 1998; 157: 1093-1097.

25. Volm M, Efferth T, Mattern J. Oncoprotein (c-myc, c-erbB1, c-erbB2, c-fos) and suppressor gene product (p53) expression in squamous cell carcinomas of the lung. Clinical and biological correlations. Anticancer Res 1992; 12: 11-20.

26. Volm M, Drings $\mathrm{P}$, Wodrich W. Prognostic significance of the expression of c-fos, c-jun and c-erbB-1 oncogene products in human squamous cell lung carcinomas. J Cancer Res Clin Oncol 1993; 119: 507510

27. Rusch V, Baselga J, Cordon-Cardo C, et al. Differential expression of the epidermal growth factor receptor and its ligands in primary non small cell lung cancers and adjacent benign lung. Cancer Res 1993; 53: 2379-2385.

28. Koukourakis MI, Giatromanolaki A, Guddo F, et al. c-erbB-2 and episialin challenges host immune response by HLA class I expression in human nonsmall-cell lung cancer. J Immunother 2000; 23: 104 114.

29. Tateishi M, Kohdono S, Hamatake M, Fukuyama $\mathrm{Y}$, Sugimachi K. Prognostic influence of the coexpression of epidermal growth factor receptor and c-erbB-2 protein in human lung adenocarcinoma. Surgical Oncol 1994; 3: 109-113.

30. Rusch V, Klimstra D, Linkov I, Dmitrovsky E. Aberrant expression of p53 or the epidermal growth factor receptor is frequent in early bronchial neoplasia and coexpression precedes squamous cell carcinoma development. Cancer Res 1995; 55: 1365-1372.

31. Meert AP, Berghmans T, Branle F, et al. Phase II and III studies with new drugs for non-small cell lung cancer: a systematic review of the literature with a methodology quality assessment. Anticancer Res 1999; 19: 4379-4390.

32. Egger M, Zellweger-Zahner T, Schneider M, Junker $\mathrm{C}$, Lengeler C, Antes G. Language bias in randomised controlled trials published in English and German. Lancet 1997; 350: 326-329.

33. Lee JS, Yoon A, Kalapurakal SK, et al. Expression of p53 oncoprotein in non-small-cell lung cancer: a favorable prognostic factor. J Clin Oncol 1995; 13: 1893-1903.

34. Stewart LA, Parmar MK. Meta-analysis of the literature or of individual patient data: is there a difference? Lancet 1993; 341: 418-422.

35. Mulrow CD. The medical review article: state of the science. Ann Intern Med 1987; 106: 485-488.

36. Meert AP, Paesmans M, Berghmans T, et al. Prophylactic cranial irradiation in small cell lung cancer: a systematic review of the literature with meta-analysis. BMC Cancer 2001; 1: 5 .

37. Auperin A, Arriagada R, Pignon JP, et al. Prophylactic cranial irradiation for patients with small-cell lung cancer in complete remission. Prophylactic Cranial Irradiation Overview Collaborative Group. N Engl J Med 1999; 341: 476-484. 